\title{
Fixed Point Theorems in 2-Metric Space for Some Contractive Conditions
}

\author{
Durdana Lateef \\ College of Science, Taibah University, Al-Madinah Al-Munawwarah, 41411, Kingdom of Saudi Arabia. \\ *Corresponding Author: Durdana Lateef, College of Science, Taibah University, Al-Madinah Al- \\ Munawwarah, 41411, Kingdom of Saudi Arabia.
}

Abstract: In this paper, we shall prove a fixed point theorem in 2-metric space by using Nesic type contractive definition. This theorem is a version of many fixed point theorem in complete metric space given by many authors announced in literature.

Keywords: Fixed point, 2-Metric spaces, Contraction, 2010 MSC: 47H10, $54 H 25$.

\section{INTRODUCTION}

The concept of fixed point theory and contraction mapping was extended and elaborated with the introduction of Contraction principle by Banach [2]. Concept of 2-metric space was introduced by Gahler [3] having the area of triangle in $\mathrm{R}^{2}$ as the inspirative example. It has been shown by Gahler that in 2-metric $\mathrm{d}$ is non-negative. After Gahler there was a flood of new results obtained by many authors in these spaces [4-8]. Military applications of fixed point theory in 2-metric spaces can be found, as well as applications in Medicine and Economics [9-11].

Then Naidu and Prasad [12] introduced the concept of weakly commuting pairs of self-mapping on a 2-metric space, then others [13] [14] and [15] have proved several common fixed point theorem by using these concept.

In this paper I proved a fixed point theorem in 2-metric space by using Nesic type contractive definition [1] and the result of Lohani and Badshah [16] also we shall use the Lemma of Singh [17].

\section{Mathematical preliminaries}

Definition 2.1 : A 2- metric space is a set $\mathrm{X}$ with non negative real Valued. function $\mathrm{d}$ on $\mathrm{X} \times \mathrm{X} \times \mathrm{X}$ satisfying the following conditions :

$\left(\mathrm{M}_{1}\right) \quad$ for two distinct point $\mathrm{x}, \mathrm{y}$ in $\mathrm{X}$ there exist a point $\mathrm{z}$

in $\mathrm{X}$ such that $\mathrm{d}(\mathrm{x}, \mathrm{y}, \mathrm{z}) \neq 0$.

$\left(\mathrm{M}_{2}\right) \quad \mathrm{d}(\mathrm{x}, \mathrm{y}, \mathrm{z})=0$ if at least two of $\mathrm{x}, \mathrm{y}, \mathrm{z}$ are equal.

$\left(\mathrm{M}_{3}\right) \quad \mathrm{d}(\mathrm{x}, \mathrm{y}, \mathrm{z})=\mathrm{d}(\mathrm{x}, \mathrm{z}, \mathrm{y})=\mathrm{d}(\mathrm{y}, \mathrm{z}, \mathrm{x})$

$\left(\mathrm{M}_{4}\right) \quad \mathrm{d}(\mathrm{x}, \mathrm{y}, \mathrm{z}) \leq \mathrm{d}(\mathrm{x}, \mathrm{y}, \mathrm{u})+\mathrm{d}(\mathrm{x}, \mathrm{u}, \mathrm{z})+\mathrm{d}(\mathrm{u}, \mathrm{y}, \mathrm{z}) \forall \mathrm{x}, \mathrm{y}, \mathrm{z}$

and $u$ in $X$.

The function $\mathrm{d}$ is called 2 metric for the space $\mathrm{X}$ and $(\mathrm{X}, \mathrm{d})$ is called 2 - metric space. "Geometrically 2 - metric represent the area".

Example : Let a mapping $d: \mathrm{R}^{3} \rightarrow[0,+\infty)$ be defined by

$d(x, y, z)=\min \{|x-y|,|y-z|,|z-x|\}$.

Then $d$ is a 2-metric on $\mathrm{R}$, i.e., the following inequality holds:

$d(x, y, z) \leq d(x, y, t)+d(y, z, t)+d(z, x, t)$,

for arbitrary real numbers $x, y, z, t$. 
Definition 2.2 :A sequence $\left\{x_{n}\right\}$ in a 2-metric space $(X, d)$ is said to be convergent to a point $\mathrm{x} \epsilon$ $\mathrm{X}$

$$
\lim _{n \rightarrow \infty} x_{n}=x, \text { if } \lim _{n \rightarrow \infty} d\left(x_{n}, x, z\right)=0 \text { for all } z \in X
$$

The point $x$ is called the limit of the sequence $\left\{x_{n}\right\}$ in $X$.

Definition 2. 3: A sequence $\left\{x_{n}\right\}$ in a 2-metric space $(X, d)$ is called a Cauchy sequence if $\lim d\left(x_{n}, x_{m}, a\right)=0$ as $n, m \rightarrow \infty$ for all a $\in X$.

Definition 2.4 : A 2-metric space ( $X, d)$ is said to be complete if every Cauchy sequence in $X$ is convergent .

Definition 2.5 : A 2-metric space ( $\mathrm{X}, \mathrm{d}$ ) is called bounded if there exist a constant $\mathrm{M}$ such that

$$
\mathrm{d}(\mathrm{x}, \mathrm{y}, \mathrm{z}) \leq \mathrm{M} \text { for all } \mathrm{x}, \mathrm{y}, \mathrm{z} \in \mathrm{X} \text {. }
$$

Definition 2.6 : A mapping $\mathrm{f}$ in 2 - metric space is called orbitally continuous if for all a in $\mathrm{X}$,

$$
\mathrm{d}\left(\mathrm{f}^{\mathrm{n}} \mathrm{x}, \mathrm{u}, \mathrm{a}\right) \rightarrow 0 \text { as } \mathrm{n} \rightarrow \infty
$$

Implies

$$
\mathrm{d}\left(\mathrm{ff}^{\mathrm{n}} \mathrm{x}, \mathrm{fu}, \mathrm{a}\right) \rightarrow 0 \text { as } \mathrm{n} \rightarrow \infty
$$

Definition 2.7: A mapping $S$ from a 2- metric space $(X, d)$ into itself is said to be sequentially continuous at a point $\mathrm{x} \in \mathrm{X}$ if every sequence $\left\{\mathrm{x}_{\mathrm{n}}\right\}$ in $\mathrm{X}$ such that

$$
\begin{aligned}
& \lim _{n \rightarrow \infty}\left(x_{n}, x, z\right)=0 \text { for all } z \in X \\
& \lim _{n \rightarrow \infty} d\left(S x_{n}, S x, z\right)=0
\end{aligned}
$$

Every convergent sequence in a 2-metric space is a cauchy sequence.

Definition 2.8: A 2 -metric space d which is continuous in all of its three arguments is called continuous.

\section{Remarks:}

(i) Every convergent sequence in 2- metric space is Cauchy.

(ii) Geometrically 2-metric space represents Area.

For proving our theorem we shall use the lemma of Singh [19].

Lemma 2.1: Let $\left\{x_{n}\right\}$ be a sequence in complete 2-metric space $X$. if there exists $h \in[0,1]$ such that

$$
\mathrm{d}\left(\mathrm{x}_{\mathrm{n}}, \mathrm{x}_{\mathrm{n}+1}, \mathrm{a}\right) \leq \mathrm{hd}\left(\mathrm{x}_{\mathrm{n}-1}, \mathrm{x}_{\mathrm{n}}, \mathrm{a}\right)
$$

for some a $€ X$ then $\left\{x_{n}\right)$ converges to point in $X$.

Drawing inspiration from Nesic type contractive definition [1] and the result of Lohani and Badshah [18], we prove the following theorem in 2-metric spaces.

\section{MAIN RESUlTS}

Theorem 2.1: Let $\mathrm{f}$ be an orbitally continuous self -map from complete 2-metric space $\mathrm{X}$ into itself, if $f$ satisfies.

$$
\begin{array}{r}
{[1+\operatorname{pd}(x, y, z) \leq p \max \{\max \{d(x, f x, a) . d(y, f y, a),} \\
d(x, f y, a) \cdot d(y, f x, a)\}
\end{array}
$$

$+q \max \{d(x, y, a), d(x, f x, a), d(y, f y, a)\}$

for all $\mathrm{x}, \mathrm{y}$ and $\mathrm{a} \in \mathrm{X}$ and $\mathrm{p} \geq 0,0<\mathrm{q}<1$, then for each $\mathrm{x} \in \mathrm{X}$,

the sequence $\left\{\mathrm{T}^{\mathrm{n}} \mathrm{x}\right\}$ converges to a unique fixed point. 
Proof: Let $\mathrm{x}_{0} \in \mathrm{X}$ be an arbitrary point and we define $\left\{\mathrm{x}_{\mathrm{n}}\right\}$ as

$$
\begin{aligned}
& x_{1}=f\left(x_{0}\right), x_{2}=f\left(x_{1}\right),-----, x_{n}=f\left(x_{n-1}\right), \ldots \ldots \ldots . . .(1.2) \\
& \text { Suppose } x_{2 n} \neq x_{2 n+1} \text { for every } x=0,1,2,---, \text { then } \\
& {\left[1+p d\left(x_{2 n}, x_{2 n+1}, a\right)\right] d\left(f\left(x_{2 n}\right), f\left(x_{2 n+1}\right), a\right)} \\
& \leq p \max \left\{d\left(x_{2 n}, f\left(x_{2 n}\right), a\right) . d\left(x_{2 n+1}, f\left(x_{2 n+1}\right), a\right),\right. \\
& \left.d\left(x_{2 n}, f\left(x_{2 n+1}\right), a\right) . d\left(x_{2 n+1}, f\left(x_{2 n}\right), a\right)\right\}+ \\
& q \text { max }\left\{d\left(x_{2 n}, x_{2 n+1}, a\right), d\left(x_{2 n}, f\left(x_{2 n}\right), a\right), d\left(x_{2 n+1}, f\left(x_{2 n+1}, a\right)\right\}\right. \\
& \text { which implies, } \\
& {\left[1+p d\left(x_{2 n}, x_{2 n+1}, a\right)\right] d\left(x_{2 n+1}, x_{2 n+2}, a\right)} \\
& \leq p \operatorname{pmax}\left\{d\left(x_{2 n}, x_{2 n+1}, a\right) \cdot d\left(x_{2 n+1}, d\left(x_{2 n+2}\right), a\right), d\left(x_{2 n}, x_{2 n+2}, a\right)\right\} \\
& +q \max \left\{d\left(x_{2 n}, x_{2 n+1}, a\right), d\left(x_{2 n}, x_{2 n+1}, a\right), d\left(x_{2 n+1}, x_{2 n+2}, a\right)\right\} \\
& d\left(x_{2 n+1}, x_{2 n+2}, a\right) \leq q \max \left\{d\left(x_{2 n}, x_{2 n+1}, a\right), d\left(x_{2 n+1}, x_{2 n+2}, a\right)\right\} \\
& \text { since } q<1,0<q /(2-q)<q, w e h a v e \\
& d\left(x_{2 n+1}, x_{2 n+2}, a\right) \leq q d\left(x_{2 n}, x_{2 n+1}, a\right)
\end{aligned}
$$

Now(1.3) hold for all a $€ \mathrm{x}$. Hence in view of lemma 2.1, the sequence $\left\{\mathrm{x}_{\mathrm{n}}\right\}$ converges to some fixed point $\mathrm{u} \in \mathrm{X}$. then for all a $\in \mathrm{X}$,

$$
\lim d\left(x_{2 n}, u, a\right)=0 \quad \text { as } n \rightarrow \infty
$$

Which implies,

$$
\lim \mathrm{d}\left(\mathrm{f}^{2 \mathrm{n}}\left(\mathrm{x}_{0}\right), \mathrm{u}, \mathrm{a}\right)=0 \text { as } \mathrm{n} \rightarrow \infty
$$

Since $\mathrm{f}$ is orbitally continuous, we have

$$
\begin{array}{llr}
\lim \mathrm{d}\left(\mathrm{f}\left(\mathrm{f}^{2 \mathrm{n}}\left(\mathrm{x}_{0}\right)\right), \mathrm{f}(\mathrm{u}), \mathrm{a}\right)=0 & \text { as } & \mathrm{n} \rightarrow \infty \\
\lim \mathrm{d}\left(\mathrm{f}^{2 \mathrm{n}+1}\left(\mathrm{x}_{0}\right), \mathrm{f}(\mathrm{u}), \mathrm{a}\right)=0 & \text { as } & \mathrm{n} \rightarrow \infty
\end{array}
$$

From the definition of 2-metric space,

$$
\begin{gathered}
\mathrm{d}(\mathrm{u}, \mathrm{f}(\mathrm{u}), \mathrm{a}) \leq \mathrm{d}\left(\mathrm{u}, \mathrm{f}(\mathrm{u}), \mathrm{f}^{2 \mathrm{n}+1}\left(\mathrm{x}_{0}\right)\right)+\mathrm{d}\left(\mathrm{u}, \mathrm{f}^{2 \mathrm{n}+1}\left(\mathrm{x}_{0}\right), \mathrm{f}(\mathrm{u})\right) \\
+\mathrm{d}\left(\mathrm{f}^{\mathrm{n}+1}\left(\mathrm{x}_{0}\right), \mathrm{f}(\mathrm{u}), \mathrm{a}\right)
\end{gathered}
$$

which tends to zero as $\mathrm{n} \rightarrow \infty$.

Consequently, $\mathrm{d}(\mathrm{u}, \mathrm{f}(\mathrm{u}), \mathrm{a})=0 \Rightarrow \mathrm{f}(\mathrm{u})=\mathrm{u}$

Uniqueness : For uniqueness of $u$, suppose $v \epsilon X$ be another common fixed point of $f$ such that $v \neq u$. hence there exists a point

$a \in X$ such that,$d(u, v, a) \neq 0$ then from (1.1) we have

$[1+P d(u, v, a)] d(f u, f v, a) \leq p \max \{d(u, f u, a) . d(v, f v, a)$,

$$
d(u, f v, a) \cdot d(v, f u, a)\}
$$

$+q \max \{d(u, v, a), d(u, f u, a), d(v, f v, a)\}$

i.e. $[1+p d(u, v, a)] d(u, v, a) \leq p \max \{d(u, u, a) \cdot d(v, v, a)$,

$$
d(u, v, a) . d(v, u, a)\}
$$

$+q \max \{d(u, v, a), d(u, u, a), d(v, v, a)\}$

$\mathrm{d}(\mathrm{u}, \mathrm{v}, \mathrm{a}) \leq \mathrm{qd}(\mathrm{u}, \mathrm{v}, \mathrm{a})$

$\mathrm{d}(\mathrm{u}, \mathrm{v}, \mathrm{a})<\mathrm{d}(\mathrm{u}, \mathrm{v}, \mathrm{a})$ which is a Contradiction.

Hence, $\mathrm{d}(\mathrm{u}, \mathrm{v}, \mathrm{a})=0$ which implies that $\mathrm{u}=\mathrm{v}$. 
The next result is the generalization of Iseki [12].

\section{Theorem 2.2:}

Let $\mathrm{f}_{1}$ and $\mathrm{f}_{2}$ be mapping of a complete bounding 2-metric space $\mathrm{X}$ into itself satisfying,

$$
\begin{aligned}
& p_{1} d\left(f_{1} x, f_{2} y, a\right)+p_{2} d\left(x, f_{1} x, a\right)+p_{3} d\left(y, f_{2} y, a\right)= \\
& \quad \min \left\{d\left(x, f_{2} y, a\right) d\left(y, f_{1} x, a\right)\right\} \leq q d(x, y, a)
\end{aligned}
$$

For all $\mathrm{x}, \mathrm{y}, \mathrm{a} \in \mathrm{X}$ there exist $\mathrm{p}_{1}, \mathrm{p}_{2}, \mathrm{p}_{3}, \mathrm{q}$ are real number such that

$$
\mathrm{p}_{1}+\mathrm{p}_{2}+\mathrm{p}_{3}>\mathrm{q}, \mathrm{q}-\mathrm{p}_{2} \geq 0, \mathrm{q}-\mathrm{p}_{3} \geq 0
$$

Then $\mathrm{f}_{1}$ and $\mathrm{f}_{2}$ have a common fixed point.

\section{Proof:}

Let $x_{0} \in X$. we define $\left\{x_{n}\right\}$

by $\mathrm{x}_{2 \mathrm{n}+1}=\mathrm{f}_{1}\left(\mathrm{x}_{2 \mathrm{n}}\right)$

$x_{2 n+2}=f_{2}\left(x_{2 n+1}\right)$

then we get,

$\mathrm{d}\left(\mathrm{x}_{\mathrm{n}}, \mathrm{x}_{\mathrm{n}+1}, \mathrm{a}\right) \leq \alpha^{\mathrm{n}} \mathrm{d}\left(\mathrm{x}_{0}, \mathrm{x}_{1}, \mathrm{a}\right)$

where $\alpha=\left(\frac{q-p 2}{p 1+p 3}\right)<1$

which implies that $\left\{x_{n}\right\}$ is Cauchy sequence and has limit say $u \in X$. Then

$\mathrm{d}\left(\mathrm{f}_{1} \mathrm{u}, \mathrm{u}, \mathrm{a}\right) \leq \mathrm{d}\left(\mathrm{f}_{1} \mathrm{u}, \mathrm{u}, \mathrm{x}_{2 \mathrm{n}+2}\right)+\mathrm{d}\left(\mathrm{f}_{1} \mathrm{u}, \mathrm{x}_{2 \mathrm{n}+2}, \mathrm{a}\right)+\mathrm{d}\left(\mathrm{x}_{2 \mathrm{n}+2}, \mathrm{u}, \mathrm{a}\right)$

from $(2.2)$

$p_{1} d\left(f_{1} a, x_{2 n+2}, a\right)+p_{2} d\left(u, T_{1}, u, a\right)+p_{3} d\left(x_{2 n+1}, x_{2 n+2}, a\right)-\min \left\{d\left(u, a_{2 n+2}, a\right)\right.$

$\left.\mathrm{d}\left(\mathrm{x}_{2 \mathrm{n}+1}, \mathrm{f}_{1} \mathrm{u}, \mathrm{a}\right)\right\} \leq \mathrm{qd}\left(\mathrm{u}, \mathrm{x}_{2 \mathrm{n}+2}, \mathrm{a}\right)$

by letting $n \rightarrow \infty$ we get

$\left(\mathrm{p}_{1}+\mathrm{p}_{2}\right) \mathrm{d}\left(\mathrm{f}_{1} \mathrm{u}, \mathrm{u}, \mathrm{a}\right) \leq 0$

We find $d\left(f_{1} u, u, q\right)=0$

for all $a \in X$. hence $f_{1} u=u$.

similarly $\mathrm{f}_{2} \mathrm{u}=\mathrm{u}$

Thus $\mathrm{u}$ is a common fixed point of $\mathrm{f}_{1}$ and $\mathrm{f}_{2}$.

\section{REFERENCES}

[1] Nesic.S.C, A theorem on contractive mappings. Mat. Vesnik 44(1992)51-54.

[2] Banach. S,: Sur les operations dans les ensembles abstraits et leur application aux equations integrals, Fund. Math. (1922), 133-181.

[3] Gahler, VS: 2-metrische Räume und ihre topologische Struktur. Math. Nachr. 26 (1963) 115-118.

[4] Cho, Y. J., Khan, M. S. and Singh, S. L. Common fixed points of weakly commuting mappings, Univ. Novomsadu, Zb. Rad. Prirod. Mt. Fak. Ser. Mat 18 (1) (1988) 129-142.

[5] Imdad, M., Khan, M. S. and Khan, M.D. A common fixed point theorem in 2-metric spaces,Math. Japonica 36 (5) (1991) 907-914.

[6] Iseki, K. A property of orbitally continuous mapping on 2-metric spaces, Math. Seminar Notes, Kobe Univ. 3(1975)131-132.

[7] Jungck, G. and Rhoades, B. E. Fixed points for set-valued function without continuity, Indian J. Pure Appl. Math., 29(1998) 227-238.

[8] Murthy, P.P., Chang, S.S., Cho, Y. J. and Sharma, B.K. Compatible mappings of type (A) and common fixed point theorems, Kyungpook Math. J., 32 (2)(1992) 203-216.

[9] Abd EL-Monsef, M. E., Abu-Donia, H. M. \& AbdRabou, Kh. New types of common fixed point theorems 2-metric spaces. Chaos, Soliton and Fractals, 41(2009) 1435-1441. 
[10] Border, K.C. Fixed point theorems with applications to economics and game theory (Cam-bridge Univ. Press, 1990).

[11] Namdeo, R.K., Dubey, S. and Tas, K. Coincidence and Fixed points of non-expansive type mappings on 2metric spaces, Mathematical Forum 2 (16) (2007) 781-789.

[12] Naidu S.V.R and Prasad J.R., Fixed point theorems in 2-metric spaces, Indian J. Pure. Appl. Math, 17(8) (1986) 974-993.

[13] Constantin, A: Common fixed points of weakly commuting mappings in 2-metric space, Math. Japon., 36(3)(1991)507-513.

[14] Iseki, K: Fixed point theorem in 2-metric space, Math. Sem., Notes, Kobe Univ., 3 (1975)133-136.

[15] Zead Mustafa, Vahid Parvaneh, Jamal Rezaei Roshan and Zoran Kadelburg, $b$-Metric spaces and some fixed point Theorems, Fixed Point Theory and Applications (2014), 144-152.

[16] Lohani P.C \& Badshah V.H, Fixed point theorem in 2- metric space. Acta Ciencia , Indica V.H., XX111(3)(1997) 243-245.

[17] S. L. Singh, "Some Contractive Type Principles on 2-Metric Spaces and Applications", Mathematics Seminar Notes (Kobe University), Vol. 7, No. 1, 1979, 1-11.

Citation: D. Lateef, " Fixed Point Theorems in 2-Metric Space for Some Contractive Conditions ", International Journal of Scientific and Innovative Mathematical Research, vol. 6, no. 1, p. 16-20, 2018., http://dx.doi.org/10.20431/2347-3142.0601003

Copyright: (C) 2018 Authors. This is an open-access article distributed under the terms of the Creative Commons Attribution License, which permits unrestricted use, distribution, and reproduction in any medium, provided the original author and source are credited. 